\title{
The development of a designated dental pathway for looked after children
}

\author{
A. Williams, ${ }^{* 1}$ J. Mackintosh, ${ }^{2}$ B. Bateman, ${ }^{2}$ S. Holland, ${ }^{1}$ A. Rushworth, ${ }^{2}$ \\ A. Brooks ${ }^{2}$ and J. Geddes ${ }^{2}$
}
IN BRIEF
- Describes the development and implementation of a dental care pathway for looked after children.
- Presents some of the challenges that providing dental care for looked after children can present.
- Acquires support for the view that the dental health of a proportion of looked after children would benefit from a care system that integrates social, medical and dental care professionals.

\begin{abstract}
Objective To explore the impact of a community-based dental care pathway on the dental care of children entering residential or foster care. Design The study used qualitative data collected during interviews with children who used the service, their carers and key professionals involved in the pathway, and routine quantitative data concerned with care entry and the dental service use. Results The dental pathway facilitated dental care access for children entering statutory care, met the dental needs of service users even when dental care provision proved challenging, and offered a consistent dental service regardless of care moves. Improved interagency integration and support was reported by key professionals as was better dissemination and documentation of dental assessments and outcomes. Conclusion The dental care pathway had a beneficial impact on the dental access and experiences of children who used it, promoted better interagency working and facilitated record keeping. These findings call for extension of the service to a wider population to allow further evaluation of its impact and efficacy in different regional areas and contexts.
\end{abstract}

\section{INTRODUCTION}

Children entering residential or foster care share many of the health problems of their peers but often to a greater level. ${ }^{1-5}$ The knowledge underpins a call for local authority and health services to work together to commission health services for all looked after children and young people (LAChildren). When considering whether dental services should be included in this call the small evidence base around the dental state of LAChildren in the UK suggests they should. Children in UK statutory care tend to have relatively high levels of poor oral care, dental neglect and disease, ${ }^{6-9}$ little regular dental attendance before care entry and higher needs for treatment when they attend a dental surgery. ${ }^{10}$

Clinical commissioning groups, local health boards and local authorities bear responsibility for providing adequate services to meet the health needs of LAChildren in England and Wales with use of primary care services encouraged. ${ }^{5}$ This

${ }^{1}$ Cardiff University School of Social Sciences, Glamorgan Building, King Edward VII Avenue

Cardiff, CF10 3WT; ${ }^{2}$ Northumbria Healthcare NHS

Foundation Trust

${ }^{*}$ Correspondence to: Dr Annie Williams

Email:WilliamsA55@cardiff.ac.uk

Online article number E6

Refereed Paper - accepted 1 November 2013

DOI: $10.1038 /$ sj.bdj.2014.51

${ }^{\circledR}$ British Dental Journal 2014; 216: E6 implies the general dental service (GDS) may be the most appropriate body to meet the dental needs of LAChildren. However, the association of LAChildren with treatment barriers, such as poor dental attendance and fear of treatment, ${ }^{11-12}$ and reports that many LAChildren suffer from significant conduct disorders ${ }^{13}$ suggests treatment within the GDS may sometimes prove challenging. An existing understanding that specialist services may be required to fully meet LAChildren's health needs (ref) turns attention to the role of community dental services (CDS) in providing dental care for LAChildren. Although a recent audit found that the majority of CDS across England and Wales were involved in the treatment of LAChildren to some extent, ${ }^{15}$ no systematic pathway to guide the dental treatment of LAChildren within the CDS appears to exist.

Designated health professionals working in a multi-agency 'Raising Health and Education of Looked After Children' (RHELAC) support team in the north of England recognised that although their local CDS treated LAChildren, the system was 'ad hoc' and could be improved. A wider group consisting of the RHELAC team and additional dental and social care professionals was formed and worked collaboratively to construct a designated dental care pathway (DDCP) for the dental care of LAChildren. The DDCP has been providing care for LAChildren since 2011.
The pilot nature of the DDCP called for an accompanying evaluation. Drawing on resultant data this paper explores the context the service was developed in, the process of the DDCP and whether the DDCP met a set of primary aims: to ensure all children entering the care system were offered dental care quickly; were offered a service that met the dental needs of children who used them in an acceptable way; whether the service impacted positively on the interagency working of key professionals involved and kept them informed of DDCP use, dental assessments and care outcomes.

\section{METHODS}

\section{Study design}

The evaluation used qualitative semistructured interviews and routine data. Ethical approval was provided by Newcastle and North Tyneside Research Ethics Committee.

\section{The intervention}

The DDCP stipulates that the dental health of children entering care is discussed at the primary medical assessment and DDCP referral offered routinely. If accepted, DCCP use is triggered by a RHELAC notification form sent to the CDS dental team. The form contains:

- LAChildren personal details

- Contact information for social workers and foster services

- Reason for care entry 


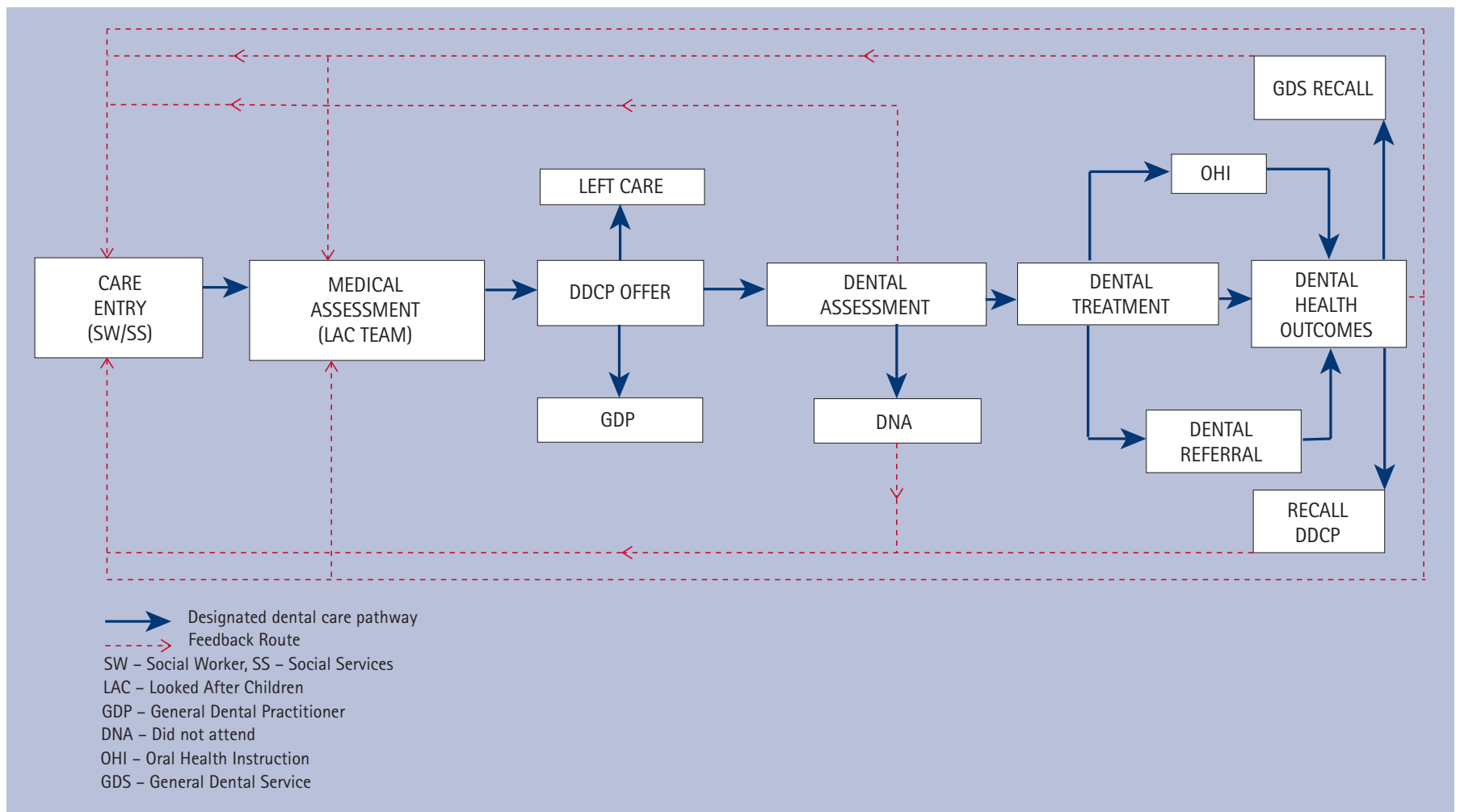

Fig. 1 The designated dental care pathway

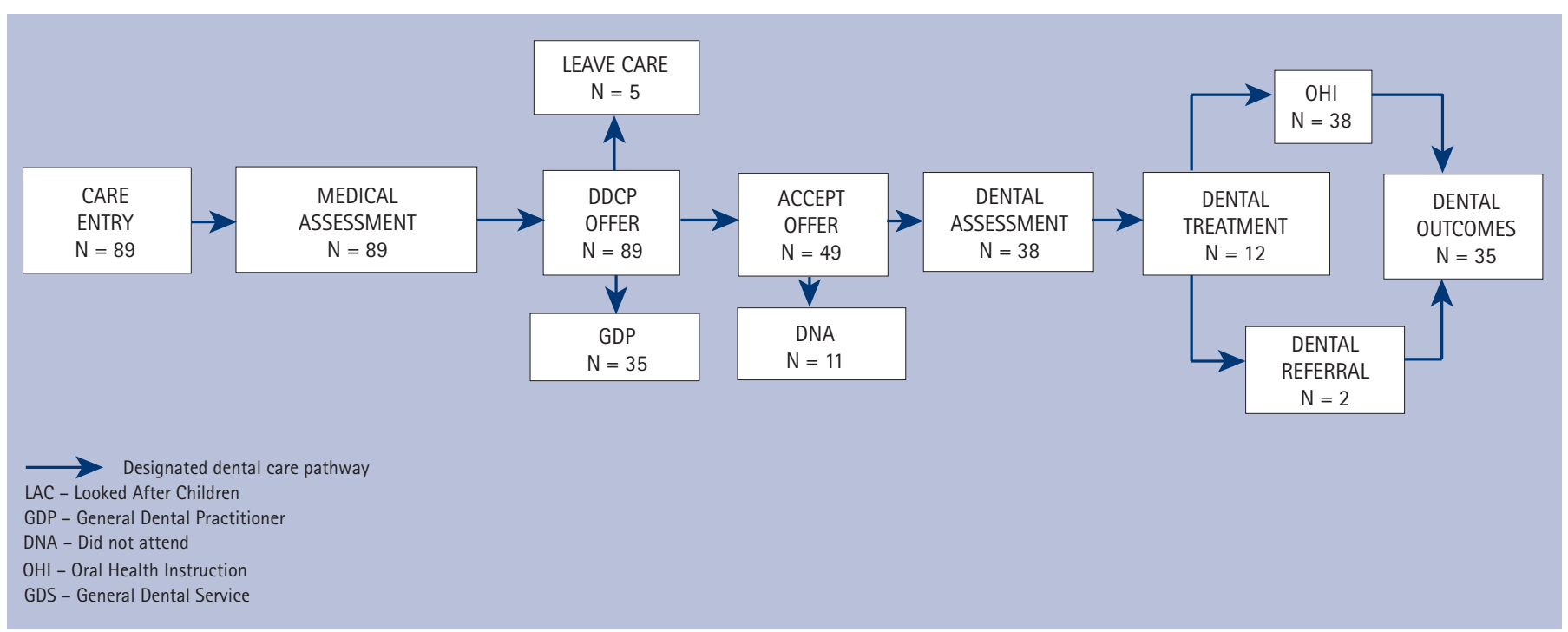

Fig. 2 Recruitment and use of the DDCP

- Consent for routine dental care from birth parent/adult with parental responsibility.

The DDCP team contacts carers, an appointment is arranged and a dental assessment conducted at a designated session at a specific CDS clinic. The resultant dental health action plan (DHAP) sets out an assessment of the child's oral health status and a dental treatment plan. Copies are forwarded to the LAChildren medical team and the child's social care team. Subsequent dental visits provide oral health sessions for all members of the foster family/residential unit, and dental treatment and/or referral to secondary dental services as required. On completion a further DHAP containing treatment details is circulated as before. A GDS or DDCP recall is organised as preferred. Figure 1 shows the pathway and its use during the evaluative period.

\section{Recruitment}

The pilot nature of the project made the potential participant group small; therefore, the resultant aim was to consult representatives of all key groups involved in the DDCP. Consequently, semi-structured interviews $(n=16)$ were conducted with: a LAChildren-designated-paediatric consultant
(DPC, $n=1$ ), a community dental officer (CDO, $\mathrm{n}=1$ ), a community dental service clinical director $(\mathrm{CD}, \mathrm{n}=1)$, a LAChildrenhealth-assessment administrator (HA, $\mathrm{n}=1$ ), an independent review chair ( $C R, n=1)$, LAChildren and foster carer social workers (SW, $\mathrm{n}=2$ ), LAChildren who used the DDCP $(n=3)$ and carers (residential carers RC, $n=2$, foster carers FC $n=3$ ). One local GDP also contributed $(n=1)$.

Professionals were approached individually, information sheets provided and written consent gained. Carers were approached during clinic visits or by letter, written information was provided and 


\begin{tabular}{|c|c|c|}
\hline DNA incident/LAC & $\mathrm{N}$ & $\%$ \\
\hline $\begin{array}{l}0 \\
1 \\
2 \\
3\end{array}$ & $\begin{array}{l}32 \\
11 \\
2 \\
4\end{array}$ & $\begin{array}{l}65.3 \\
22.4 \\
4.1 \\
8.2\end{array}$ \\
\hline Total & 49 & 100 \\
\hline
\end{tabular}

Table 2 Number of treatment appointments

\begin{tabular}{l|l|l}
$\begin{array}{l}\text { Treatment } \\
\text { appointments (n) }\end{array}$ & LAC (n) & Total \\
\hline 0 & 26 & 0 \\
1 & 4 & 4 \\
2 & 4 & 8 \\
3 & 3 & 9 \\
4 & 1 & 4 \\
5 & 1 & 5 \\
6 & 1 & 6 \\
\hline Total & 36 & 36
\end{tabular}

written consent gained before interviews. LAChildren were approached through carers who discussed involvement, gained consent and arranged the interviews.

\section{Data collection}

Routine data collected during statutory care entry and DDCP use gave information about the numbers of children who:

- Entered residential or foster care in the area served by the DDCP over a 12 month period (2011-2012)

- Were offered, referred to and used the DDPC.

Oral health promotion specialists conducted face-to-face interviews either in the professionals' places of work or in the homes of LAChildren and carers. All interviews were digitally recorded.

\section{Data analysis}

Interviews were transcribed, anonymised and entered into password-protected university files. Analysis using NVivo 9.2 (qualitative research software) identified the following themes around which an analytic framework-matrix was organised:

- The dental health of LAChildren on entering care

- Dental treatment for LAChildren before the DDPC

- The experience and receipt of the DDCP

- The impact of the DDCP on interagency working and knowledge.

Quantitative data was entered into SPSS 18 (predictive analytics software) to gain descriptive statistics of the DDCP take up and the children who used the service.

\section{RESULTS}

Over a 12 month period 89 children and young people entered care and were offered DDCP referral. Forty-nine accepted, 35 intended to visit a GDP and five left care (Fig. 2).

The 49 referred children were 0-17 years of age: 24 male, 24 female (1, no response). Forty-four (89\%) LAChildren were white (British/other), two African, and one European, (two no response).

Eleven of the 49 children who made appointments for a DDCP assessment did not attend (DNA). Table 1 shows a number of the 39 children who used the DDCP, DNA'd on at least one occasion. Twelve children required further dental treatment. Table 2 shows this took between $1-6$ visits.

Qualitative analysis collected information about the dental health of LAChildren in the area served by the DDCP before care entry and their dental treatment options pre-DDCP. It also explored the provision and receipt of DDCP and its impact on interagency working.

\section{The dental context}

The dental health of LAChildren on care entry

A history of poor dental attendance, hygiene and poor diet was reported by experienced social workers, residential and foster carers.

Many LAChildren had little experience of dental attendance or a record of irregular attendance before care entry: ' $a$ lot of them didn't have any [dentist]' (RC1), 'although they had been on the roll of a local dentist they hadn't been for some time' (RC2). Poor attendance contributed to high levels of anxiety and appointment refusals: 'some would rather put up with toothache than go to the dentist' (CR).

Poor oral hygiene was also noted: 'reflecting perhaps their neglected circumstances, they presented with much worse oral hygiene than their peers' (SW1), with some children having little experience of tooth cleaning at all: 'I've done lots of littlies, the younger children, and sometimes they've never had a toothbrush never mind anything else...with the older ones it's even harder, trying to establish a routine they've never had' (FC1).

Many older LAChildren displayed poor attitudes to oral health: 'ranges from really poor to OK... we get very few young people who come into our care with really positive messages around dental health care' (RC1).

Other threats such as a poor diet: 'they would eat sweets, crisps...no the diets are very poor...vegetables, most of the children that come to me could only identify maybe two vegetables' (FC3), and prolonged use of dummies/bottles: 'the oldest one? Seven, with a dummy!' (FC3) were identified.

\section{Dental pathways pre-DDCP}

Many LAChildren used the GDS, but for some accessing dental care through this route provide difficult or impossible.

Some LAChildren continued to attend family dentists, although travelling or potential contact with birth parents could prevent this: 'they don't want to go back to the family dentist in case there are problems with parents coming across them' (CD).

If no family dentist existed or was inaccessible carers had to seek treatment, usually within the NHS. This was possible if 'foster carers have built up a good relationship with their professionals, so you'll find the dentists are very tolerant of LAChildren' (SW1). Otherwise no system to ensure NHS access existed: 'I had to say "ahh you [carer] ring NHS direct and see if you can get a dentist"' (HA).

There was some history of GDPs referring LAChildren into the CDS: 'sometimes foster children were referred [to CDS] by various dentists' (CDO).

The time needed to treat LAChildren with behavioural or emotional difficulties appeared to be a barrier to accessing treatment in the GDS: 'the children have quite complex needs, they can at times be quite challenging, quite disturbed' (RC1). To complicate this, underlying difficulties were sometimes sensitive and difficult to address: 'I brushed against his face as you do in clinical work, and he completely flinched. It was only at this point we started talking, it was an indicator of the abusive relationship he had with his father' (CD). Time was also a problem when treatment needs were high: 'you can't justify having the child for a long time' (GDP).

When appointments had been booked a late cancellation or failure to attend often ensued: 'they haven't been to the dentist for a long time...then they are suddenly faced with a dental appointment, and often they are fine, and then the day before or the day of the appointment, they categorically refuse to go' (RC1).

Experiences of LAChildren being de-registered were common: 'we have had dentists who have terminated people because they have not turned up for one appointment or they have turned up and they felt their behaviour is not appropriate for a dentist' (RC1).

\section{The DDCP}

\section{DDCP experiences}

The DDCP was valued for accessibility, expertise and flexibility. 
The link between medical and dental services allowed quick access 'in the past there have been gaps of 2 or 3 months... and now we can get that service almost immediately, it's made a huge difference' (RC2).

CDS staff were experienced in treating patients with additional needs: 'our clinicians are incredibly skilled at getting all sorts of people to do all sort of things, because they take a lot of time and they have built up a relationships over a long time' (CD), and possessed knowledge of LAChildren's needs: 'they're used to dealing with the type of children we have, because these aren't ordinary children. These aren't run of the mill, like sort of cross section [sic]. These are normally very damaged' (FC3).

DDCP resources allowed additional/ prolonged appointments if necessary: 'that's, to us, a major advantage to have somewhere like that rather than a really busy dental surgery' (RC2). The success of this was demonstrated by treatment completion: 'I have not had any of the LAChildren actually who have not really stuck with me and got something done' (CDO).

The DDCP had resources which coped with missed/cancelled appointments 'the communication has been absolutely brilliant. Obviously, we try and let them know if young people are not going to attend, but that can be a very short space of time beforehand and there has been massive understanding around that' (RC1). DDCP staff have visited non-attending LAChildren to make contact, describe the service and alleviate anxiety. The DDCP also provides care continuity if LAChildren change placements or return home '[and] continued to access care here' (CDO).

Concerns about the DDCP sessions being seen as stigmatising proved unfounded: 'there is always this thing about stigma with LAChildren, but no one is going to know they go to a specialist LAChildren dentist, it's not really the kind of thing children talk about' (SW2), as did anxiety that travel difficulties to the clinic may prevent use. Although some respondents thought GDS treatment may be perceived as more normal and therefore preferable, it was felt that this was not possible for all at present: 'you would hope that every LAChild could attend a GDP like everyone else... and the service would be really sensitive and responsive to their needs. But we are not there. That's not the case' (RC1).

There was some feeling the DDCP benefited carers 'it's one less thing for them to worry about and sort out themselves when they have so many things to do' (CDO). Carers gave good feedback 'the young people who have gone up there have been treated with respect...and they received extremely good dental care as well' (RC2). LAChildren echoed this: 'I would go every minute!' (LAC1), 'Some people would just do stuff but she is very caring when she is going to do stuff to your teeth. The rest of the team, they are just the same' (LAC2).

\section{The impact of the DDCP on interagency working}

The DDCP appeared to improve interagency working and the dissemination of dental health assessments/outcomes.

Dental staff could contact medical and/or social workers: 'I have got other people to go to if they do miss appointments or I have concerns about their dental health' (CDO).

The DHAP form seemed to have improved interagency awareness of dental service use, although a couple reports indicated there was some need for improvement: 'I know they have been to the dentist and whether they needed treatment or not. But no one said to me that they have been to this service' (CR).

Feeding dental records back into the social care system appeared to help social care professional keep better records about whether statutory demands were being met: 'there is an administrative advantage in terms of a targeted service... a greater ability to monitor attendance, monitor healthcare, oral care' (SW1).

Professionals appreciated having a support system for wider concerns: 'Having clinical relationships with a designated nurse and doctor means if the dental team have any wider concerns for child they have an immediate source of help and advice our staff have someone to go to, somebody specific who has knowledge and expertise' (CD).

\section{DISCUSSION}

This study adds to the limited knowledge about the dental health of LAChildren in the UK and, to our knowledge is the first to explore the work of a dental system tailored to the needs of LAChildren.

The study adds to evidence that suggests LAChildren often enter care with little experience of receiving regular dental care and with existing dental needs, ${ }^{10-12}$ and furthermore, indicates the oral health of LAChildren is often compromised by poor oral hygiene, histories of unhealthy diets and prolonged use of bottles and dummies.

The study also suggests that although many LAChildren receive dental assessment and care through the GDS, access and use of the GDS can be dependent on a carer's resources and the needs and behaviours of the LAChildren. The possibility that dental care within the GDS cannot be found for some LAChildren cannot be discounted. This situation calls for alternative more systematic routes, such as the DDCP, that ensure all children can access dental treatment when they enter care and during their time in care.

Overall, the DDCP ensured quick access to a dental team used to working with patients with additional needs, it also led to good levels of treatment completion which demonstrated the value of specialist health services to meet the health needs of LAChildren when needed. ${ }^{14}$

The ability of the DDCP feedback loop to identify and disseminate incidents of LAChildren non-attendance was useful in facilitating follow up by dental and/or social teams, although gaps within the system after a DNA became obvious and efforts to remedy them are needed. Despite this, the DDCP generally provided continuity of dental care for LAChildren using the service, even after residence changes which are often barriers to health care access for LAChildren. ${ }^{16}$ DDCP produced high levels of satisfaction among LAChildren and their carers.

The DDCP was established through relatively minor, mostly administrative changes which integrated existing services to produce a system which ensured access to NHS dental treatment, and enabled all concerned with the child's care to be kept informed of service use and outcomes. This reinforces opinion that LAChildren services require good design, integration and information sharing between all involved ${ }^{17}$ and calls for consideration of whether the DDCP model could be extended to GDS where no system to disseminate records of LAChildren's dental treatment exists.

Most study limitations grew from the study size and its pilot nature. Cautious interpretation of the low percentage of LAChildren requiring dental treatment $(34 \%)$ is advised as the some of the areas study children were raised in are artificially fluoridated water ${ }^{18}$ which could have affected dental disease levels found. The small size of the sample also raises questions about travel and access to the DDCP which appeared unproblematic in this case but may cause problems elsewhere.

\section{CONCLUSION}

This study supports evidence that many LAChildren have dental needs on statutory care entry, and suggests that accessing care within the GDS can be difficult at present. The DDCP was able to offer LAChildren quick access to comprehensive, consistent dental care, and ensure feedback about the child's dental service use and outcomes to all key professionals. The lack of a similar system elsewhere calls for a wider trial 
within the CDS in England and Wales and consideration of extending the system into the GDS.

The work was undertaken with the support of The Centre for the Development and Evaluation of Complex Interventions for Public Health Improvement (DECIPHer), a UKCRC Public Health Research: Centre of Excellence. DECIPHer gratefully acknowledges funding from the British Heart Foundation, Cancer Research UK, Economic and Social Research Council (RES-590-28-0005), Medical Research Council, the Welsh Government and the Wellcome Trust (WT087640MA), under the auspices of the UK Clinical Research Collaboration.

1. Mooney A, Statham J, Monck E, Chambers H. Promoting the health of looked after children: a study to inform revision of the 2002 guidance. Research Report DCSF-RR125. Department for Children, Schools and Families, 2009

2. Hochstadt N, Jaudes P. Zimo D, Schachter J. The medical and social needs of children entering foster care. Child Abuse Neg/ 1987; 11: 53-62.

3. Kerker B, Dore M. Mental health needs and treatment of foster youth: Barriers and opportunities. Am J Orthopsychiatry 2006; 76: 138-147.

4. Ward H, Lynch M, Skuse $T$, Jones H. Issues concerning the health of looked after children. Adopt Foster J 2002; 26: 8-18.

5. Department of Schools, Families and Children. Statutory guidance on promoting the health and wellbeing of looked after children. Department of
Schools, Families and Children, 2009. Online report available at http://media.education.gov.uk/assets/ files/pdf/s/promotinghealth.pdf (accessed November 2013).

6. Waddell B. The dental health of looked after and accommodated children and young people in Scotland - a literature review. University of Glasgow, 2008. Online review available at http://hdl.handle. net/1905/735 (accessed November 2013).

7. Poynor M, Welbury J. The dental health of looked after children. Adopt Foster 2004; 28: 86-88.

8. Scott J, Hill M. The health of looked after and accommodated children and young people in Scotland - messages from research. Edinburgh: Social Work Inspection Agency, 2006. Online report available at http://www. scotland.gov.uk/Publications/2006/06/07103730/0 (accessed November 2013).

9. Sarri G, Evans P, Stansfeld S, Marcenes W. A schoolbased epidemiological study of dental neglect among adolescents in a deprived area of the UK. $\mathrm{Br}$ Dent J 2012; 10: E17.

10. Williams J, Jackson S, Maddocks A, Cheung W-Y, Love A, Hutchings $\mathrm{H}$. Case control study of the health of those looked after by authorities. Arch Dis Childhood 2001; 5: 280-285.

11. Herrera M. Molinero C. Missed appointments at a NHS dental practice. Universitat Politecnica de Catalunya, 2004. Online report available at http:// upcommons.upc.edu/e-prints/bitstream/2117/304/1/ dentistenglish 1.pdf (accessed November 2013).

12. Nuttall N, Freeman R, Beavan-Seymour C, Hill K Access and barriers to care: a report for the Adult dental health Survey. Leeds: The Information Centre for
Health and Social Care, 2011. Online report available at http://www.dhsspsni.gov.uk/theme8_barriersandaccesstocare.pdf (accessed November 2013).

13. Melzer H, Corbin T, Gatward R, Goodman R, Ford T. The mental health of young people looked after by local authorities in England. London: The Stationery Office, 2003.

14. National Institute for Health and Care Excellence (NICE). Quality standard for the health and wellbeing of looked after children and young people. NICE OS31. Manchester: NICE, 2013. Online information available at http://publications.nice.org.uk/quality-standard-forthe-health-and-wellbeing-of-looked-after-childrenand-young-people-qs31/introduction-and-overview (accessed November 2013).

15. Collard M, Leck R, Viles A, Williams A. Community Dental Service: An audit of dental treatment pathways for Looked After Children in England and Wales. DF2 audit: Newcastle University and Cardiff University. In preparation.

16. Jones R, Everson-Hock E, Guillaume L et al. The effectiveness of interventions aimed at improving access to health and mental health services for looked after children and young people. Families, Relationships \& Societies 2012; 1: 71-85.

17. Social Care Institute for Excellence. SCIE research briefing 9: preventing teenage pregnancy in lookedafter children. London: Social Care Institute for Excellence, 2005

18. The British Fluoridation Society. One in a million: the facts about water fluoridation. Online information available at http://www.bfsweb.org/onemillion/onemillion2012.html (accessed November 2013). 